\title{
Cytisine for smoking cessation
}

\author{
Taleen Karnieg PharmD, Xiang Wang PharmD MSc
}

Cite as: CMAJ 2018 May 14;190:E596. doi: 10.1503/cmaj.171371

\section{1}

\section{Cytisine mimics the effects of nicotine}

Cytisine, primarily derived from the Cytisus laburnum plant (which inspired the development of varenicline), is a partial agonist of the $\alpha_{4} \beta_{2}$ nicotinic acetylcholine receptor responsible for central effects of nicotine. ${ }^{1}$ Oral cytisine reaches peak concentration two hours postdose ${ }^{2}$ and is excreted unchanged renally without hepatic metabolism, lowering the risk of drug interactions. ${ }^{2}$ Oral cytisine has a shorter half-life (4.8 v. $17 \mathrm{~h}$ ) and treatment course ( $3.5 \mathrm{v} .12 \mathrm{wk}$ ) than varenicline. ${ }^{2,3}$

\section{Cytisine is not a new drug}

Cytisine has been safely used in eastern Europe for smoking cessation since the $1960 \mathrm{~s} .{ }^{1,3}$ Its generic structure renders market exclusivity difficult, and its low selling price offers little financial incentive for companies to seek costly regulatory approval elsewhere. ${ }^{3}$ Contraindications include arterial hypertension, advanced atherosclerosis, and pregnancy or breastfeeding. ${ }^{4,5}$ These were based on exclusion criteria from cytisine trials, which were derived from contraindications of nicotine products. ${ }^{4,5}$ The adverse effects reported with cytisine are largely gastrointestinal (dyspepsia and nausea) and sleep disturbance, which are self-limiting. ${ }^{1,3-5}$

\section{3}

\section{Cytisine is effective for smoking cessation}

A systematic review and meta-analysis of eight controlled trials involving 4020 adult smokers reported a 59\% $(p<0.00001)$ higher abstinence rate from smoking while taking cytisine versus placebo, with a number needed to treat (NNT) of 6.35. ${ }^{6}$ An open-label, randomized trial $(n=1310)$ showed a higher self-reported abstinence rate at one month on cytisine versus nicotine replacement therapy among adult daily smokers $(40 \% \mathrm{v}$. $31 \%$, respectively; $p<0.001$; NNT 11). ${ }^{5}$ Similarly, the self-reported abstinence rate at six months was higher among participants taking cytisine compared with nicotine replacement therapy ( $22 \%$ v. $15 \%$, respectively; $p=0.002$; NNT 14$).{ }^{5}$

\section{Cytisine is available in Canada without a prescription}

- Cytisine was approved by Health Canada in August 2017 as a natural health product. Compared with prescription medications with a drug identification number, natural health products have less stringent regulatory approval requirements regarding the burden of scientific evidence for efficacy and safety. Patients can purchase cytisine without a prescription from select pharmacies, health shops and directly online from the manufacturer. Cytisine has a complex dosing regimen (Box 1$).^{5}$

\section{Cytisine is cost-effective}

A report by the Canadian Agency for Drugs and Technologies in Health estimates the cost of smoking cessation treatment in Canada as follows: nicotine replacement therapy $(\$ 360-\$ 440)$, bupropion $(\$ 215)$ and varenicline $(\$ 340) .^{7}$ The current price of cytisine is $\$ 56.49$ for the 25 -day treatment course.

\section{Box 1: Cytisine dosing regimen}

\begin{tabular}{ll} 
Days & \multicolumn{1}{c}{ Dosing regimen } \\
\hline $1-3$ & 1 capsule every $2 \mathrm{~h}$ (maximum: 6 capsules/d) \\
\hline $4-12$ & 1 capsule every $2.5 \mathrm{~h}$ (maximum: 5 capsules/d) \\
\hline $13-16$ & 1 capsule every $3 \mathrm{~h}$ (maximum: 4 capsules/d) \\
\hline $17-20$ & 1 capsule every 5 h (maximum: 3 capsules/d) \\
\hline $21-25$ & $1-2$ capsules/d
\end{tabular}

\section{References}

1. Rigotti NA. Cytisine - a tobacco treatment hiding in plain sight. N Engl J Med 2014;371:2429-30.

2. Jeong SH, Newcombe D, Sheridan J, et al. Pharmacokinetics of cytisine, an $\alpha 4 \beta 2$ nicotinic receptor partial agonist, in healthy smokers following a single dose. Drug Test Anal 2015;7:475-82.

3. Walker N, Bullen C, Barnes J, et al. Getting cytisine licensed for use world-wide: a call to action. Addiction 2016;111:1895-8.

4. West R, Zatonski W, Cedzynska M, et al. Placebo-controlled trial of cytisine for smoking cessation. N Engl J Med 2011;365: 1193-200.

5. Walker N, Howe C, Glover M, et al. Cytisine versus nicotine for smoking cessation. N Engl J Med 2014;371:2353-62.

6. Hajek P, McRobbie H, Myers K. Efficacy of cytisine in helping smokers quit: systematic review and meta-analysis. Thorax 2013;68:1037-42.

7. Drugs for smoking cessation: information for health professionals. Ottawa: Canadian Agency for Drugs and Technologies in Health; 2016. Available: www.cadth.ca/sites/default/files/pdf/ drugs_for_smoking_cessation.pdf (accessed 2017 Nov. 11).

Competing interests: Taleen Karnieg and Xiang Wang have no financial or business relationships with manufacturers of pharmaceutical preparations of cytisine or pharmacies stocking these products. They did not receive any compensation for the writing of this article.

This article has been peer reviewed.

Affiliations: Leslie Dan Faculty of Pharmacy (Karnieg, Wang), University of Toronto, Toronto, Ont.; Department of Pharmacy (Wang), The Ottawa Hospital, Ottawa, Ont.

Correspondence to: Xiang Wang,wanqus@gmail.com 